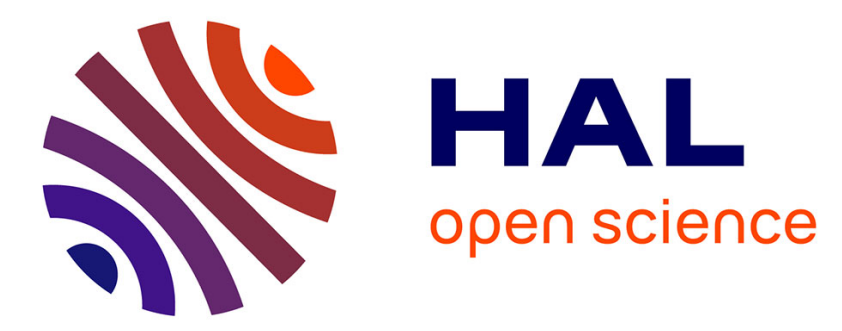

\title{
Comparison principle and Liouville type results for singular fully nonlinear operators.
}

\author{
Isabeau Birindelli, Françoise Demengel
}

\section{To cite this version:}

Isabeau Birindelli, Françoise Demengel. Comparison principle and Liouville type results for singular fully nonlinear operators.. Annales de Faculté des sciences de Toulous, 2004, 13 (2), pp.261-287. hal-00842591

\section{HAL Id: hal-00842591 \\ https://hal.science/hal-00842591}

Submitted on 9 Jul 2013

HAL is a multi-disciplinary open access archive for the deposit and dissemination of scientific research documents, whether they are published or not. The documents may come from teaching and research institutions in France or abroad, or from public or private research centers.
L'archive ouverte pluridisciplinaire HAL, est destinée au dépôt et à la diffusion de documents scientifiques de niveau recherche, publiés ou non, émanant des établissements d'enseignement et de recherche français ou étrangers, des laboratoires publics ou privés. 


\title{
Comparison principle and Liouville type results for singular fully nonlinear operators.
}

\author{
I. Birindelli \\ Universita di Roma la sapienza, instituto Guido Castelnuovo \\ F. Demengel \\ Université de Cergy-Pontoise, AGM UMR 8088
}

\section{Introduction}

This paper is two folded : On one hand, we prove a comparison result for singular fully nonlinear operators modeled on the $p$-Laplacian. In the second part we use this and a strong maximum principle to obtain Liouville type results.

The solutions considered are taken in the viscosity sense even though the standard definitions need to be adapted to our singular operators. As in the work of Evans and Spruck [9] and the work of Juutinen, Lindquist, Manfredi [14], we take into account that we cannot take test functions whose gradient is zero in the test point since the operator may not be defined when this occurs.

We shall consider an operator $F$ defined on $\mathbb{R}^{N} \times\left(\mathbb{R}^{N}\right)^{\star} \times S$, where $S$ denotes the space of symmetric matrices on $\mathbb{R}^{N}, F$ is continuous. $F$ is supposed to satisfy some of the following conditions :

1. $F(x, p, 0)=0, \forall x, p \in \mathbb{R}^{N} \times\left(\mathbb{R}^{N}\right)^{\star}$.

2. There exists a continuous function $\omega, \omega(0)=0$, such that if $(X, Y) \in S^{2}$ and $\zeta$ satisfy

$$
-\zeta\left(\begin{array}{cc}
I & 0 \\
0 & I
\end{array}\right) \leq\left(\begin{array}{cc}
X & 0 \\
0 & Y
\end{array}\right) \leq 4 \zeta\left(\begin{array}{cc}
I & -I \\
-I & I
\end{array}\right)
$$


and $I$ is the identity matrix in $\mathbb{R}^{N}$, then for all $(x, y) \in \mathbb{R}^{N}$,

$$
F(x, \zeta(x-y), X)-F(y, \zeta(x-y),-Y) \leq \omega\left(\zeta|x-y|^{2}\right) .
$$

3. $\exists \alpha, \beta \in \mathbb{R}^{2}, \alpha \geq \beta>-1,(\lambda, \Lambda) \in\left(\mathbb{R}^{+}\right)^{2}$, such that $\forall(x, p, M, N) \in$ $\mathbb{R}^{N} \times\left(\mathbb{R}^{N}\right)^{\star} \times S^{2}, N \geq 0$

$$
|p|^{\beta} \lambda \operatorname{tr} N \leq F(x, p, M+N)-F(x, p, M) \leq\left(\frac{|p|^{\alpha}+|p|^{\beta}}{2}\right) \Lambda \operatorname{tr} N
$$

Let us note that among the functions satisfying all the conditions above there is the function

$$
F(p, M)=|p|^{\alpha} \mathcal{M}_{\lambda, \Lambda}^{+} M
$$

where $\mathcal{M}_{\lambda, \Lambda}^{+} M=\Lambda \sum_{e_{i}>0} e_{i}+\lambda \sum_{e_{i}<0} e_{i}$ and $e_{1}, . . e_{N}$ are the eigenvalues of $M$ (see Caffarelli - Cabrè [5] ). Other examples, including the $p$-Laplacian, are given at the beginning of section 2 .

Of course condition 3 implies the monotonicity of $F$ i.e. in particular that $\forall(x, p, M, N) \in \mathbb{R}^{N} \times\left(\mathbb{R}^{N}\right)^{\star} \times S^{2}, N \geq 0$

$$
0 \leq F(x, p, M+N)-F(x, p, M) .
$$

Furthermore if $F$ does not depend explicitly on $x$, condition 2 is not necessary.

In his famous work [12] Jensen proved comparison results for viscosity solutions of

$$
F(u, \nabla u, \nabla \nabla u)=0
$$

for a class of $F$ everywhere defined. This was a crucial step in the development of viscosity theory for second order elliptic operators (see e.g. Ishii, JensenLions-Souganidis, Crandall Lions, etc, [11], [13], [7]).

In the sequel we shall define the concept of viscosity solutions for inequations of the form

$$
F(x, \nabla u, \nabla \nabla u)-g(x, u) \geq 0(\leq 0)
$$

where $g$ is supposed to be continuous on $\mathbb{R}^{N} \times \mathbb{R}$. Moreover in Theorem 1.1, we shall establish some comparison principle when $g(x, u)=b(u)$ where $b$ is a continuous function on $\mathbb{R}$ which is non-decreasing and such that $b(0)=0$.

In the first part, our main result is the following 
Theorem 1.1 Let $\Omega$ be a bounded open set in $\mathbb{R}^{N}$. Suppose that $F$ satisfies conditions 1, 2, and 3. Suppose that $b$ is some continuous and increasing function on $\mathbb{R}$, such that $b(0)=0$. Suppose that $u \in \mathcal{C}(\bar{\Omega})$ is a viscosity sub-solution of $F=b$ and $v \in \mathcal{C}(\bar{\Omega})$ is a viscosity supersolution of $F=b$ :

If $u \leq v$ on $\partial \Omega$, then $u \leq v$ in $\Omega$.

If $b$ is nondecreasing, the same result holds when $v$ is a strict supersolution or vice versa when $u$ is a strict subsolution.

Let us remark that the super and sub-solutions are taken in the sense given in Definition 2.1 below.

This result implies, of course, uniqueness of viscosity solutions for Dirichlet problems in bounded domains. Furthermore it allows us to prove a strong maximum principle when $b$ is zero (Proposition 2.2). The case where $b$ is non zero but satisfies some increasing behavior at infinity is treated in [18].

The proof of Theorem 1.1 follows the strategy of the proof of comparison theorems for second order elliptic operators without singularities which doubles the variables and uses a technical Lemma due to Jensen (see Lemma 2.3 below). Here two difficulties arise, the first is due to the fact that we can't use functions with gradient equal to zero at the test points, hence we need to prove explicitly that this is not the case. Secondly condition 3 requires the tests functions to be constructed with functions modeled on $\psi(x)=b+a|x|^{q}$ with $q>\frac{\alpha+2}{\alpha+1}$, therefore Jensen's lemma cannot be used as is, we need to prove some other ad hoc technical Lemma (see Lemma 2.1 below).

Let us also remark that our proof doesn't differentiate the case $\alpha>0$ (where the operator is degenerate elliptic) and $\alpha<0$ where the operator is singular.

In the second part we consider the inequation

$$
\left\{\begin{array}{l}
-F\left(x, \nabla u, D^{2} u\right) \geq h(x) u^{\bar{q}} \text { in } \mathbb{R}^{N} \\
u \geq 0
\end{array}\right.
$$

where $F$ is a continuous function satisfying conditions 1,3 . Condition 3 will be assumed with $\alpha=\beta$; furthermore $h$ is a smooth function such that $h(x) \geq C|x|^{\gamma}$ for $|x|$ large and for some $\gamma$ that will be specified later.

Let us observe that for $\alpha=0$ and $\lambda=\Lambda=1$ the above equation becomes 


$$
\left\{\begin{array}{l}
-\Delta u \geq h(x) u^{\bar{q}} \text { in } \mathbb{R}^{N} \\
u \geq 0 .
\end{array}\right.
$$

In this case Gidas in [10] and Berestycki, Capuzzo-Dolcetta, Nirenberg [1] proved, for classical solutions, that when $1<\bar{q} \leq \frac{N+\gamma}{N+\gamma-2}$ there are no nontrivial solutions. This result is optimal, in the sense that for any $\bar{q}>\frac{N+\gamma}{N+\gamma-2}$ it is possible to construct a non trivial positive $\mathcal{C}^{2}$ solution of equation (1.1) (see [4]).

The main result in the second part is the following

Theorem 1.2 Suppose that $F$ satisfies condition 1,3. Suppose that $u \in$ $\mathcal{C}\left(\mathbb{R}^{N}\right)$ is a nonnegative viscosity solution of

$$
-F\left(x, \nabla u, D^{2} u\right) \geq h(x) u^{\bar{q}} \text { in } \mathbb{R}^{N}
$$

with h satisfying

$$
h(x)=a|x|^{\gamma} \text { for }|x| \text { large, } a>0 \text { and } \gamma>-(\alpha+2) .
$$

Let $\mu=\frac{\Lambda}{\lambda}(N-1)-1$. Suppose that

$$
0<\bar{q} \leq \frac{1+\gamma+(\alpha+1)(\mu+1)}{\mu}
$$

then $u \equiv 0$.

When $\alpha=0$, for standard viscosity solutions, this result is due to Cutrì and Leoni [8].

When $F$ is in a class of divergence form operators (including the $p$ Laplacian) this result was obtained by Mitidieri and Pohozaev [15] using integral estimates that cannot be applied in our case.

The value $\frac{1+\gamma+(\alpha+1)(\mu+1)}{\mu}$ is equal to $\frac{N+\gamma}{N+\gamma-2}$ when $\lambda=\Lambda$ and $\alpha=\beta=0$ i.e. the case of the Laplacian. 


\section{Comparison principles}

Before stating the comparison principle, let us make a few remarks and give some examples about operators satisfying conditions 1,2 and 3 .

Remark 2.1 It is quite standard to see that condition 3 implies that $\forall(x, p, M, N) \in \mathbb{R}^{N} \times\left(\mathbb{R}^{N}\right)^{\star} \times S^{2}$,

$$
\begin{aligned}
|p|^{\beta} \lambda t r N^{+}-\left(|p|^{\alpha}+|p|^{\beta}\right) \frac{\Lambda}{2} \operatorname{tr} N^{-} & \leq F(x, p, M+N)-F(x, p, M) \\
& \leq\left(|p|^{\alpha}+|p|^{\beta}\right) \frac{\Lambda}{2} \operatorname{tr} N^{+}-|p|^{\beta} \lambda t r N^{-}
\end{aligned}
$$

where $N=N^{+}-N^{-}$is a minimal decomposition of $N$ into the difference of two nonnegative matrices. This of course implies that for $\alpha=\beta$ :

$$
|p|^{\alpha} \mathcal{M}_{\lambda, \Lambda}^{-}(N) \leq F(x, p, N) \leq|p|^{\alpha} \mathcal{M}_{\lambda, \Lambda}^{+}(N)
$$

where $\mathcal{M}_{\lambda, \Lambda}^{-}(N)$ and $\mathcal{M}_{\lambda, \Lambda}^{+}(N)$ are the so called Pucci operators defined by

$$
\mathcal{M}_{\lambda, \Lambda}^{-}(N)=\lambda\left(\sum_{e_{i}>0} e_{i}\right)+\Lambda\left(\sum_{e_{i}<0} e_{i}\right), \mathcal{M}_{\lambda, \Lambda}^{+}(N)=\lambda\left(\sum_{e_{i}<0} e_{i}\right)+\Lambda\left(\sum_{e_{i}>0} e_{i}\right)
$$

where the $e_{i\{1 \leq i \leq N\}}$ are the eigenvalues of $N$.

Example 2.1 Evans and Spruck in [9] have considered the evolution of level sets by mean curvature i.e. they have studied:

$$
u_{t}=\left(\delta_{i j}-\frac{u_{x_{j}} u_{x_{i}}}{|D u|^{2}}\right) u_{x_{i}, x_{j}}
$$

in $\mathbb{R}^{N} \times[0,+\infty)$.

Let us remark that the associated stationary operator:

$$
F(p, N)=\operatorname{tr} N-\frac{(N p, p)}{|p|^{2}}
$$

satisfies the assumptions 1,3. (See also the work of Chen, Giga and Goto [6]). 
Example 2.2 In the case of the q-Laplacian, 3 is satisfied with $\beta=\alpha=$ $q-2$. Indeed the $q$-Laplacian is defined by

$$
F(p, N)=|p|^{q-2} \operatorname{tr} N+(q-2)|p|^{q-4}(N p, p) .
$$

Example 2.3 Let us consider

$$
F(p, N)=\frac{\sqrt{|p|^{8}+2|p|^{6}+3|p|^{4}+1}}{|p|^{\frac{3}{4}}} \operatorname{tr} N+b(N p, p)
$$

with $b \geq 0$. Then 3 is satisfied with $\alpha=\frac{13}{4}$ and $\beta=-\frac{3}{4}$.

We now present an example where $F$ depends explicitly on $x$.

Example 2.4 Suppose that $q_{1}, q_{2}$ are real numbers such that $1<q_{1} \leq 2$, $1<q_{2} \leq 2, c\left(q_{1}, q_{2}\right)$ is such that

$$
c\left(q_{1}, q_{2}\right)\left\{\begin{array}{cc}
>0 & \text { if } q_{1} \neq q_{2} \\
\geq q_{1}-2 & \text { if } q_{1}=q_{2}
\end{array}\right.
$$

and suppose that $B_{1}$ and $B_{2}$ are two Lipschitz functions which send $\bar{\Omega}$ into $S$. Then the function

$$
F(x, p, N)=|p|^{q_{1}-2} \operatorname{tr}\left(B_{1}^{\star}(x) B_{1}(x) N\right)+c\left(q_{1}, q_{2}\right)|p|^{q_{2}-4}\left(N\left(B_{2}(x) p, B_{2}(x) p\right)\right)
$$

satisfies conditions 1,2, 3.

Indeed conditions 1 to 3 are immediate, we shall prove condition 2 . In a first time we check that when $B$ is a matrix with Lipschitz coefficients and $1<q_{1}<2$, the operator

$$
|p|^{q_{1}-2} \operatorname{tr}\left(B^{\star}(x) B(x) N\right)
$$

satisfies 2 . For that aim let $X, Y$, such that

$$
\left(\begin{array}{cc}
X & 0 \\
0 & Y
\end{array}\right) \leq \zeta\left(\begin{array}{cc}
I & -I \\
-I & I
\end{array}\right)
$$

Then for $\xi, \eta \in \mathbb{R}^{N}$ we use the inequality

$$
(X \xi, \xi)+(Y \eta, \eta) \leq \zeta|\xi-\eta|^{2}
$$


with $\xi=B(x) e_{i}$ and $\eta=B(y) e_{i}$ and $e_{i}$ is some vector of the canonical basis.

$$
\begin{aligned}
\left(X B(x) e_{i}, B(x) e_{i}\right) & +\left(Y B(y) e_{i}, B(y) e_{i}\right) \\
& \leq \zeta_{j}\left|(B(x)-B(y)) e_{i}\right|^{2} \\
& \leq \zeta_{j}|x-y|^{2}(\operatorname{Lip} B)^{2}\left|e_{i}\right|^{2} .
\end{aligned}
$$

Summing over $i=1,2, . . N$ one gets

$$
\begin{aligned}
F\left(x, \zeta_{j}(x-y), X\right)-F\left(y, \zeta_{j}(x-y),-Y\right) & \leq c\left(\zeta_{j}\left|x_{j}-y_{j}\right|\right)^{q_{1}-2}\left(\zeta_{j}|x-y|^{2}\right) \\
& \left.=\left(\zeta_{j}|x-y|^{2}\right)^{q_{1}-1}|x-y|^{2-q_{1}}\right) \\
& \leq(\operatorname{diam} \Omega)^{2-q_{1}}\left(\zeta_{j}|x-y|^{2}\right)^{q_{1}-1}
\end{aligned}
$$

which goes to zero with $\zeta_{j}|x-y|^{2}$, since $1<q_{1}<2$.

We now treat the second term

$$
\begin{aligned}
(X B(x) p, B(x) p)+(Y B(y) p, B(y) p) & \leq \zeta_{j}|B(x) p-B(y) p|^{2} \\
& \leq(\operatorname{Lip} B)^{2} \zeta_{j}|x-y|^{2}|p|^{2}
\end{aligned}
$$

Using this with $p=\zeta_{j}(x-y)$ one obtains

$$
\begin{aligned}
|p|^{q_{2}-4}((X B(x) p, B(x) p)+(Y B(y) p, B(y) p)) & \leq \zeta_{j}|x-y|^{2}\left(\zeta_{j}|x-y|\right)^{q_{2}-2} \\
& =\left(\zeta_{j}|x-y|^{2}\right)^{q_{2}-1}|x-y|^{2-q_{2}}
\end{aligned}
$$

this goes to zero when $\left(\zeta_{j}|x-y|^{2}\right)$ does, since $\left.\left.q_{2} \in\right] 1,2\right]$.

Before introducing viscosity solutions in this setting, we want to prove a weak maximum principle for classical $C^{2}$ solutions:

Proposition 2.1 Let $\Omega$ be a bounded open set in $\mathbb{R}^{N}$. Suppose that $b$ is some non decreasing continuous function on $\mathbb{R}$, such that $b(0)=0$. Suppose that $u$ is $\mathcal{C}^{2}(\Omega)$ and satisfies

$$
F\left(x, \nabla u, D^{2} u\right)-b(u) \leq 0 \text { in } \Omega
$$

where $F$ satisfies 1 and the left hand side of $3, u \geq 0$ on $\partial \Omega$. Then $u \geq 0$ inside $\Omega$. 
Proof.

Suppose by contradiction that $u$ has a strictly negative minimum. Let $x_{0} \in \Omega$, such that $u\left(x_{0}\right)<0$, and $\epsilon<\frac{-u\left(x_{0}\right)}{\operatorname{diam}(\Omega)^{2}}$. Then the function $u_{\epsilon}(x)=u(x)-$ $\frac{\epsilon}{2}\left|x-x_{0}\right|^{2}$ also has a strictly negative minimum which is achieved inside $\Omega$. Indeed if one supposes that it is achieved on the boundary, say at $x_{\epsilon} \in \partial \Omega$, then

$$
u_{\epsilon}\left(x_{\epsilon}\right)=u\left(x_{\epsilon}\right)-\frac{\epsilon}{2}\left|x_{\epsilon}-x_{0}\right|^{2} \geq \frac{u\left(x_{0}\right)}{2}>u\left(x_{0}\right)=u_{\epsilon}\left(x_{0}\right)
$$

a contradiction.

At the point $x_{\epsilon}$ one has

$$
D^{2} u\left(x_{\epsilon}\right) \geq \epsilon I
$$

and then, even if $D u\left(x_{\epsilon}\right)=0$, one can find a point $x_{\epsilon}^{\prime}$ around $x_{\epsilon}$ such that $D u\left(x_{\epsilon}^{\prime}\right) \neq 0$, and $D^{2} u\left(x_{\epsilon}^{\prime}\right) \geq \frac{\epsilon}{2}$. Using this and the fact that $b\left(u\left(x_{\epsilon}^{\prime}\right)\right) \leq 0$, the inequality in 3 becomes

$$
\begin{aligned}
0 & \geq F\left(x_{\epsilon}^{\prime}, D u\left(x_{\epsilon}^{\prime}\right), D^{2} u\left(x_{\epsilon}^{\prime}\right)\right)-b\left(u\left(x_{\epsilon}^{\prime}\right)\right) \\
& \geq \lambda\left|D u\left(x_{\epsilon}^{\prime}\right)\right|^{\beta} \epsilon N \\
& >0
\end{aligned}
$$

which is a contradiction.

In the definition below, $g$ denotes some continuous function defined on $\mathbb{R}^{N} \times \mathbb{R}$.

Definition 2.1 Let $\Omega$ be an open set in $\mathbb{R}^{N}$, then $v \in \mathcal{C}(\Omega)$ is called a viscosity super-solution of $F=g(x,$.$) if for all x_{0} \in \Omega$,

-Either there exists an open ball $B\left(x_{0}, \delta\right), \delta>0$ in $\Omega$ on which $v=$ cte $=c$ and $g(x, c) \geq 0$

-Or $\forall \varphi \in \mathcal{C}^{2}(\Omega)$, such that $v-\varphi$ has a local minimum on $x_{0}$ and $D \varphi\left(x_{0}\right) \neq 0$, one has

$$
F\left(x_{0}, D \varphi\left(x_{0}\right), D^{2} \varphi\left(x_{0}\right)\right) \leq g\left(x_{0}, v\left(x_{0}\right)\right) .
$$

Of course $u$ is a viscosity sub-solution if for all $x_{0} \in \Omega$, 
-Either there exists a ball $B\left(x_{0}, \delta\right), \delta>0$ on which $u=$ cte $=c$ and $g(x, c) \leq$ 0 ,

-Or $\forall \varphi \in \mathcal{C}^{2}(\Omega)$, such that $u-\varphi$ has a local maximum on $x_{0}$ and $D \varphi\left(x_{0}\right) \neq 0$, one has

$$
F\left(x_{0}, D \varphi\left(x_{0}\right), D^{2} \varphi\left(x_{0}\right)\right) \geq g\left(x_{0}, u\left(x_{0}\right)\right) .
$$

We shall say that $v$ is a strict super-solution (respectively $u$ is a strict subsolution) if there exists $\epsilon>0$ such that for all $x_{0} \in \Omega$, either there exists an open ball $B\left(x_{0}, \delta\right), \delta>0$ in $\Omega$ on which $v=$ cte $=c$ and $g(x, c) \geq \epsilon$, or $\forall \varphi \in \mathcal{C}^{2}(\Omega)$, such that $v-\varphi$ has a local minimum on $x_{0}$ and $D \varphi\left(x_{0}\right) \neq 0$, one has

$$
F\left(x_{0}, D \varphi\left(x_{0}\right), D^{2} \varphi\left(x_{0}\right)\right) \leq g\left(x_{0}, v\left(x_{0}\right)\right)-\epsilon .
$$

(respectively either $u=$ cte on a ball $B\left(x_{0}, \delta\right)$ and $g(x$, cte $) \leq-\epsilon$, or in (2.2), one has $F\left(x_{0}, D \varphi\left(x_{0}\right), D^{2} \varphi\left(x_{0}\right)\right) \geq g\left(x_{0}, u\left(x_{0}\right)\right)+\epsilon$.)

Remark 2.2 When $g \equiv 0$ the conditions on locally constant solutions are automatically satisfied for sub or super solutions.

Theorem 2.1 Let $\Omega$ be a bounded open set in $\mathbb{R}^{N}$. Suppose that $F$ satisfies condition 1, 2, 3, that $b$ is some increasing continuous function on $\mathbb{R}$, such that $b(0)=0$. Assume that $u \in \mathcal{C}(\bar{\Omega})$ is a viscosity sub-solution of $F=b($. and $v \in \mathcal{C}(\bar{\Omega})$ is a viscosity super-solution of $F=b($.$) , and that u \leq v$ on $\partial \Omega$, then $u \leq v$ in $\Omega$.

If $b$ is nondecreasing the same result holds when $v$ is a strict supersolution or vice versa when $u$ is a strict subsolution.

For convenience we start by recalling the definition of semi-jets given in [7] (see also [11], page 140,)

$$
\begin{aligned}
J^{2,+} u(\bar{x}) & =\left\{(p, X) \in \mathbb{R}^{N} \times S, u(x) \leq u(\bar{x})+\langle p, x-\bar{x}\rangle+\right. \\
& \left.\left.+\frac{1}{2}\langle X(x-\bar{x}), x-\bar{x})\right\rangle+o\left(|x-\bar{x}|^{2}\right)\right\}
\end{aligned}
$$

and

$$
\begin{aligned}
J^{2,-} u(\bar{x}) & =\left\{(p, X) \in \mathbb{R}^{N} \times S, u(x) \geq u(\bar{x})+\langle p, x-\bar{x}\rangle+\right. \\
& \left.+\frac{1}{2}\langle X(x-\bar{x}), x-\bar{x})\right\rangle+o\left(|x-\bar{x}|^{2}\right\}
\end{aligned}
$$


Clearly when $(p, X) \in J^{2,+} u(\bar{x})$ and $p \neq 0$ the function $\phi(x)=u(\bar{x})+\langle p, x-$ $\left.\bar{x}\rangle+\frac{1}{2}\langle X(x-\bar{x}), x-\bar{x})\right\rangle$ will be a test function for $u$ at $\bar{x}$ if $u$ is a subsolution.

Before starting the proof we state the analogous of the famous standard result (see e.g. Lemma 1 in Ishii [11]) used in comparison theorems for second order equations:

Lemma 2.1 Let $\Omega$ be a bounded open set in $\mathbb{R}^{N}$. Let $u \in \mathcal{C}(\bar{\Omega}), v \in \mathcal{C}(\bar{\Omega})$, $\left(x_{j}, y_{j}\right) \in \Omega^{2}, x_{j} \neq y_{j}$, and $q \geq 3$.

We assume that the function

$$
\psi_{j}(x, y)=u(x)-v(y)-\frac{j}{q}|x-y|^{q}
$$

has a local maximum on $\left(x_{j}, y_{j}\right)$, with $x_{j} \neq y_{j}$. Then, there are $X_{j}, Y_{j} \in \mathcal{S}^{N}$ such that

$$
\begin{gathered}
\left(j\left(\left|x_{j}-y_{j}\right|^{q-2}\left(x_{j}-y_{j}\right), X_{j}\right) \in J^{2,+} u\left(x_{j}\right)\right. \\
\left(j\left(\left|x_{j}-y_{j}\right|^{q-2}\left(x_{j}-y_{j}\right),-Y_{j}\right) \in J^{2,-} v\left(y_{j}\right)\right.
\end{gathered}
$$

and

$$
-4 j k_{j}\left(\begin{array}{cc}
I & 0 \\
0 & I
\end{array}\right) \leq\left(\begin{array}{cc}
X_{j} & 0 \\
0 & Y_{j}
\end{array}\right) \leq 3 j k_{j}\left(\begin{array}{cc}
I & -I \\
-I & I
\end{array}\right)
$$

where

$$
k_{j}=2^{q-3} q(q-1)\left|x_{j}-y_{j}\right|^{q-2} .
$$

We postpone the proof of Lemma 2.1, but let us just remark that since $\left(\begin{array}{cc}I & -I \\ -I & I\end{array}\right)$ annihilates vectors of type $\left(\begin{array}{c}x \\ x\end{array}\right)$, then $X_{j} \leq-Y_{j}$.

Proof of Theorem 2.1

Suppose by contradiction that $\max (u-v)>0$ in $\Omega$. Let us consider for $j \in \mathbb{N}$ and for some $q>\max \left(2, \frac{\alpha+2}{\alpha+1}\right)$

$$
\psi_{j}(x, y)=u(x)-v(y)-\frac{j}{q}|x-y|^{q}
$$


Suppose that $\left(x_{j}, y_{j}\right)$ is a maximum for $\psi_{j}$. Extracting a subsequence still denoted $\left(x_{j}, y_{j}\right)$, one has $\left(x_{j}, y_{j}\right) \rightarrow(\bar{x}, \bar{y})$ for some $(\bar{x}, \bar{y}) \in \bar{\Omega}^{2}$.

Furthermore from

$$
\psi_{j}\left(x_{j}, y_{j}\right) \geq \psi_{j}\left(x_{j}, x_{j}\right),
$$

one obtains that $j\left|x_{j}-y_{j}\right|^{q} \leq C$, hence $\bar{x}=\bar{y} \in \bar{\Omega}$.

On the other hand

$$
\begin{aligned}
u(\bar{x})-v(\bar{x}) & \geq \varlimsup \\
& \geq \varlimsup_{\lim } \psi_{j}\left(x_{j}\right)-v\left(x_{j}, y_{j}\right) \\
& \geq \varlimsup_{x \in \Omega} \psi_{j}(x, x) \\
& \geq \sup _{x \in \Omega}(u(x)-v(x))
\end{aligned}
$$

and $\bar{x}$ is a point where $(u-v)$ achieves its maximum. In the same time we have obtained that $j\left|x_{j}-y_{j}\right|^{q} \rightarrow 0$.

Claim: For $j$ large enough $x_{j} \neq y_{j}$.

We first remark using

$$
\psi_{j}\left(x_{j}, x_{j}\right) \geq \psi_{j}\left(x_{j}, x\right)
$$

and

$$
\psi_{j}\left(x_{j}, x_{j}\right) \geq \psi_{j}\left(x, x_{j}\right)
$$

that $x_{j}$ is a maximum (respectively minimum) point for $x \mapsto u(x)+\frac{j}{q}\left|x-x_{j}\right|^{q}$ (respectively $\left.x \mapsto v(x)+\frac{j}{q}\left|x-x_{j}\right|^{q}\right)$.

Next, we observe that one can assume that these maximum and minimum are both strict.

Indeed, suppose for example that the minimum is not strict for $x \mapsto$ $v(x)+\frac{j}{q}\left|x-x_{j}\right|^{q}$, then, there exist $\delta>0$ and $R>\delta$ such that $B\left(x_{j}, R\right) \subset \Omega$ and

$$
v\left(x_{j}\right)=\inf _{\delta \leq\left|x-x_{j}\right| \leq R}\left\{v(x)+\frac{j}{q}\left|x-x_{j}\right|^{q}\right\} .
$$

Hence, if $y_{j}$ is a point on which the minimum above is achieved, one has

$$
v\left(x_{j}\right)=v\left(y_{j}\right)+\frac{j}{q}\left|x_{j}-y_{j}\right|^{q},
$$


and $\left(x_{j}, y_{j}\right)$ is still a maximum point for $\psi_{j}$ since

$$
u\left(x_{j}\right)-v\left(y_{j}\right)-\frac{j}{q}\left|x_{j}-y_{j}\right|^{q}=u\left(x_{j}\right)-v\left(x_{j}\right) \geq u(x)-v(y)-\frac{j}{q}|x-y|^{q} .
$$

In this case the claim is proved.

We now assume that $x_{j}$ is a strict minimum for $x \mapsto v(x)+\frac{j}{q}\left|x-x_{j}\right|^{q}$ and $x_{j}$ is a strict maximum for $x \mapsto u(x)+\frac{j}{q}\left|x-x_{j}\right|^{q}$.

We define $Z^{+}=\{z \in \mathbb{R}, b(z)>0\}$ and $Z^{-}=\{z \in \mathbb{R}, b(z)<0\}$.

We shall now prove that

-Either $b$ is increasing, and $v\left(x_{j}\right) \geq 0, u\left(x_{j}\right) \leq 0$.

-Or $b$ is increasing and

If $u$ is a strict subsolution, $u\left(x_{j}\right) \in Z^{-}, v\left(x_{j}\right) \in \mathbb{R} \backslash Z^{-}$.

If $v$ is a strict supersolution, $v\left(x_{j}\right) \in Z^{+}, u\left(x_{j}\right) \in \mathbb{R} \backslash Z^{+}$.

In particular one can note that when $b=0$ this cannot happen and the claim is proved.

In each of the cases one gets a contradiction with $u\left(x_{j}\right)>v\left(x_{j}\right)$, since $b$ is nondecreasing.

To prove this we shall use the following lemma whose proof will be given later :

Lemma 2.2 Let $v$ be a continuous, viscosity supersolution of

$$
F(x, \nabla v(x), \nabla \nabla v(x))-b(v(x)) \leq-\epsilon_{1}
$$

with $\epsilon_{1} \geq 0$ for all $x$ in $\Omega$. Suppose that $\bar{x}$ is some point in $\Omega$ such that

$$
v(x)+C|x-\bar{x}|^{q} \geq v(\bar{x}),
$$

where $\bar{x}$ is a strict local minimum of the left hand side and $v$ is not locally constant around $\bar{x}$. Then,

$$
b(v(\bar{x})) \geq \epsilon_{1}
$$

Remark 2.3 Of course the analogous result is true for subsolutions.

Suppose first that $b$ is increasing :

If $v$ is locally constant, by definition $b\left(v\left(x_{j}\right)\right) \geq 0$, and $v\left(x_{j}\right) \geq 0$. In the same manner if $u$ is locally constant $u\left(x_{j}\right) \leq 0$. We finally assume that nor 
$u$ neither $v$ is locally constant. Then, applying Lemma 2.2 to $u$ and $v$ with $\epsilon_{1}=0, C=\frac{j}{q}$ and $\bar{x}=x_{j}$, one obtains that $b\left(v\left(x_{j}\right)\right) \geq 0$, and $b\left(u\left(x_{j}\right)\right) \leq 0$, which imply that $u\left(x_{j}\right) \leq 0$, and $v\left(x_{j}\right) \geq 0$.

We now consider the case where $b$ is non decreasing, and suppose that $v$ is a strict super-solution. If $v$ is locally constant around $x_{j}$ one gets $b\left(v\left(x_{j}\right)\right)>0$, hence $v\left(x_{j}\right) \in Z^{+}$. If $v$ is not locally constant, using lemma 2.2 one gets $b\left(v\left(x_{j}\right)>0\right.$. On the other hand if $u$ is locally constant around $x_{j}$, by defintion $b\left(u\left(x_{j}\right)\right) \leq 0$, and if $u$ is not locally constant using lemma 2.2 for $u$ one gets that $b\left(u\left(x_{j}\right)\right) \leq 0$. Similarly if $u$ is a strict subsolution and $v$ is a supersolution one obtains that $b\left(u\left(x_{j}\right)<0, b\left(v\left(x_{j}\right) \geq 0\right.\right.$. We have then obtained the desired result.

We now conclude. Let $\epsilon>0$ be given. Suppose first that $b$ is increasing. Since $u(\bar{x})-v(\bar{x})=m>0$, one can take $j$ large enough in order that

$$
b\left(u\left(x_{j}\right)\right)-b\left(v\left(x_{j}\right)\right) \geq \frac{\epsilon}{4} \text { and } \omega\left(j\left|x_{j}-y_{j}\right|^{q}\right) \leq \frac{\epsilon}{4} .
$$

Then using Lemma 2.1, and property 2 and 3 of $F$, one gets

$$
\begin{aligned}
0 & \leq F\left(x_{j}, j\left|x_{j}-y_{j}\right|^{q-2}\left(x_{j}-y_{j}\right), X_{j}\right)-b\left(u\left(x_{j}\right)\right) \\
& \leq F\left(x_{j}, j\left|x_{j}-y_{j}\right|^{q-2}\left(x_{j}-y_{j}\right), X_{j}\right)-b\left(v\left(y_{j}\right)\right)-\frac{\epsilon}{4} \\
& \leq F\left(y_{j}, j\left|x_{j}-y_{j}\right|^{q-2}\left(x_{j}-y_{j}\right),-Y_{j}\right)-b\left(v\left(y_{j}\right)\right)-\frac{\epsilon}{4} \\
& +\omega\left(j\left|x_{j}-y_{j}\right|^{q}\right) \\
& \leq \frac{-\epsilon}{2} .
\end{aligned}
$$

In the case where $b$ is nondecreasing let $\epsilon$ be given such that

$$
F(x, \nabla v, \nabla \nabla v)-b(v(x)) \leq-\epsilon
$$

then take $j$ large enough in order that

$$
\omega\left(j\left|x_{j}-y_{j}\right|^{q}\right) \leq \frac{\epsilon}{2} .
$$

One has, using Lemma 2.1, property 2 and 3 of $F$ and the nondecreasing behavior of $b$,

$$
0 \leq F\left(x_{j}, j\left|x_{j}-y_{j}\right|^{q-2}\left(x_{j}-y_{j}\right), X_{j}\right)-b\left(u\left(x_{j}\right)\right)
$$




$$
\begin{aligned}
& \leq F\left(x_{j}, j\left|x_{j}-y_{j}\right|^{q-2}\left(x_{j}-y_{j}\right), X_{j}\right)-b\left(v\left(y_{j}\right)\right) \\
& \leq F\left(y_{j}, j\left|x_{j}-y_{j}\right|^{q-2}\left(x_{j}-y_{j}\right),-Y_{j}\right)-b\left(v\left(y_{j}\right)\right) \\
& +\omega\left(j\left|x_{j}-y_{j}\right|^{q}\right) \\
& \leq \frac{-\epsilon}{2} .
\end{aligned}
$$

In both cases, one gets a contradiction and it ends the proof of Theorem 2.1.

Proof of Lemma 2.2:

Without loss of generality we can assume that $\bar{x}=0$. Let $m_{\delta}$ be defined as

$$
m_{\delta}=\inf _{\delta \leq|x| \leq R}\left\{v(x)+C|x|^{q}\right\}>v(0)
$$

and

$$
\epsilon=m_{\delta}-v(0)
$$

We choose $N_{o}$ large enough in order to have $N_{0}>\frac{1}{\delta}$ and $N_{0}>\frac{4 q(\operatorname{diam} \Omega)^{q-1} C}{\epsilon}$ and such that for $|x-y| \leq \frac{1}{N_{0}}$, one has

$$
|v(x)-v(y)|+|b(v(x))-b(v(y))| \leq \frac{\epsilon}{4} .
$$

Since $v$ is not locally constant and $q>1$ for all $n$ there exists $\left(t_{n}, z_{n}\right) \in$ $B\left(0, \frac{1}{n}\right)$ with

$$
v\left(z_{n}\right)+C\left|z_{n}-t_{n}\right|^{q}<v\left(t_{n}\right) .
$$

We prove that for $n \geq N_{o}$

$$
\inf _{|x| \leq \delta}\left(v(x)+C\left|x-t_{n}\right|^{q}\right)<\inf _{\delta \leq|x| \leq R}\left(v(x)+C\left|x-t_{n}\right|^{q}\right) .
$$

Indeed

$$
\begin{aligned}
\inf _{|x| \leq \delta}\left(v(x)+C\left|x-t_{n}\right|^{q}\right) & \leq v\left(z_{n}\right)+C\left|z_{n}-t_{n}\right|^{q} \\
& <v\left(t_{n}\right) \\
& \leq v(0)+\frac{\epsilon}{4}
\end{aligned}
$$


On the other hand, for $n>N_{o}$ :

$$
\begin{aligned}
\inf _{R \geq|x| \geq \delta}\left(v(x)+C\left|x-t_{n}\right|^{q}\right) & \geq \inf _{x}\left(v(x)+C|x|^{q}+C\left|x-t_{n}\right|^{q}-C|x|^{q}\right) \\
& \geq \epsilon+v(0)-q C\left|t_{n}\right|(\operatorname{diam} \Omega)^{q-1} \\
& \geq v(0)+\frac{3 \epsilon}{4}
\end{aligned}
$$

Finally the minimum is achieved in $B(0, \delta)$.

Moreover, using (2.3), the point on which the minimum is achieved is not $t_{n}$, hence the function

$$
\varphi_{n}(x)=-C\left|x-t_{n}\right|^{q}
$$

is a test function for $v$ on a point $z_{\delta}^{n}$. Using the property 3 of $F$, one obtains that for some constant $C^{\prime}$

$$
\left|F\left(z_{\delta}^{n}, \nabla \varphi_{n}\left(z_{\delta}^{n}\right), \nabla \nabla \varphi_{n}\left(z_{\delta}^{n}\right)\right)\right| \leq C^{\prime}|\delta|^{q(\alpha+1)-(\alpha+2)} .
$$

Consequently, since $q>\frac{\alpha+2}{\alpha+1}$, we can choose $\delta$ such that $C^{\prime} \delta^{q(\alpha+1)-(\alpha+2)} \leq$ $\frac{\epsilon}{4}$ and then

$$
\begin{aligned}
b(v(0)) & \geq b\left(v\left(z_{\delta}^{n}\right)\right)-\frac{\epsilon}{8} \\
& \geq b\left(v\left(z_{\delta}^{n}\right)\right)-F\left(z_{\delta}^{n}, \nabla \varphi_{n}\left(z_{\delta}^{n}\right), \nabla \nabla \varphi_{n}\left(z_{\delta}^{n}\right)\right)-\frac{\epsilon}{4} \\
& \geq \epsilon_{1}-\frac{\epsilon}{4}
\end{aligned}
$$

This ends the proof, since $\epsilon$ is arbitrary.

Proof of Lemma 2.1.

The proof is a consequence of two technical facts and a lemma which is the analogous of Lemma 9 in Ishii [11].

Claim 1 Let $A_{j}$ be defined as

$$
A_{j}=j\left(\begin{array}{cc}
D_{j} & -D_{j} \\
-D_{j} & D_{j}
\end{array}\right)
$$


where $D_{j}=2^{q-3} q C_{j}$ and $C_{j}=\left|x_{j}-y_{j}\right|^{q-2}\left(I+(q-2)\left(x_{j}-y_{j}\right) \otimes\left(x_{j}-y_{j}\right)\right)$. Then

$$
A_{j}+\frac{1}{j} A_{j}^{2} \leq 2 j\left\|D_{j}\right\|\left(\begin{array}{cc}
I & -I \\
-I & I
\end{array}\right) .
$$

\section{Claim 2}

Suppose that $q>2$. Then

$$
\frac{1}{q}|\xi+\eta|^{q}-\frac{1}{q}-<\xi, \eta>-2^{q-3} q\left(|\eta|^{2}+(q-2)<\xi, \eta>^{2}\right) \leq 0
$$

for any $\xi \neq 0, \xi \in \mathbb{R}^{N},|\xi|=1$ and $\eta \in \mathbb{R}^{N}$ such that

$$
|\xi| \geq|\eta|
$$

Lemma 2.3 Let $(u, v) \in U S C\left(\mathbb{R}^{N}\right)$ and $A \in S^{2 N}$, and assume that $u(0)=$ $v(0)=0$ and

$$
u(x)+v(y) \leq(x, y) A\left(\begin{array}{l}
x \\
y
\end{array}\right)
$$

for all $x, y \in \mathbb{R}^{N}$ then for all $\epsilon>0$ there are $(X, Y) \in S^{N}$ such that

$$
(0, X) \in J^{2,+}(u(0)),(0, Y) \in J^{2,-}(v(0))
$$

and

$$
-\left(\frac{1}{\epsilon}+\|A\|\right)\left(\begin{array}{cc}
I & 0 \\
0 & I
\end{array}\right) \leq\left(\begin{array}{cc}
X & 0 \\
0 & Y
\end{array}\right) \leq A+\epsilon A^{2} .
$$

The proof of this lemma can be found in Ishii [11], while the proofs of the claims are given below.

We are now able to prove Lemma 2.1. We use the arguments in Ishii [11]. Let $j$ be large enough in order to have $\left|x_{j}-y_{j}\right|<\frac{1}{j\left|x_{j}-y_{j}\right|^{q-1}+1}$. For $\epsilon$ small enough, $\epsilon \leq \frac{\left|x_{j}-y_{j}\right|}{2}$, define $u_{j}$ and $v_{j} \in U S C\left(\mathbb{R}^{N}\right)$

$$
u_{j}(x)=\mid \begin{array}{cc}
u\left(x+x_{j}\right)-u\left(x_{j}\right)-j\left|x_{j}-y_{j}\right|^{q-2}<x_{j}-y_{j}, x>, & |x| \leq \epsilon \\
\frac{-|x|^{2}}{\epsilon^{3}}-2|| u \|_{\infty}, & |x|>\epsilon
\end{array}
$$




$$
v_{j}(y)=\mid \begin{array}{cc}
-v\left(y+y_{j}\right)+v\left(y_{j}\right)+j\left|x_{j}-y_{j}\right|^{q-2}<x_{j}-y_{j}, y>, & |y| \leq \epsilon \\
\frac{-|y|^{2}}{\epsilon^{3}}-2\|v\|_{\infty}, & |y|>\epsilon
\end{array}
$$

We need to prove that these functions are USC. Starting with $u$ one must check that for $|x|=\epsilon$

$$
\frac{-|x|^{2}}{\epsilon^{3}}-2|| u \|_{\infty} \leq u\left(x+x_{j}\right)-u(x)-j\left|x_{j}-y_{j}\right|^{q-2}<x_{j}-y_{j}, x>.
$$

This is satisfied since $\epsilon<\left|x_{j}-y_{j}\right|$ implies

$$
\epsilon^{2} \leq\left|x_{j}-y_{j}\right|^{2} \leq \frac{1}{j\left|x_{j}-y_{j}\right|^{q-2}+1} \leq \frac{1}{j\left|x_{j}-y_{j}\right|^{q-1}+1} .
$$

For $v$ we must check that for $|y|=\epsilon$ :

$$
-2\|v\|_{\infty}-\frac{|y|^{2}}{\epsilon^{3}} \leq-v\left(y+y_{j}\right)+v\left(y_{j}\right)+j\left|x_{j}-y_{j}\right|^{q-2}<x_{j}-y_{j}, y>
$$

which is satisfied since

$$
\epsilon^{2} \leq \frac{1}{j\left|x_{j}-y_{j}\right|^{q-1}+1}
$$

In order to apply Lemma 2.3. we need to prove that for $A_{j}$ as defined in claim 1 ,

$$
u_{j}(x)+v_{j}(y) \leq(x, y) A_{j}\left(\begin{array}{l}
x \\
y
\end{array}\right) .
$$

For that aim we distinguish several cases :

First case. Suppose that $|x| \leq \epsilon$ and $|y| \leq \epsilon$. Then $|x-y| \leq 2 \epsilon \leq$ $\left|x_{j}-y_{j}\right|$.

We prove then that for $|x-y| \leq\left|x_{j}-y_{j}\right|$

$$
u_{j}(x)+v_{j}(y) \leq(x, y) A_{j}\left(\begin{array}{l}
x \\
y
\end{array}\right) .
$$

Indeed one has

$$
(x, y) A_{j}\left(\begin{array}{l}
x \\
y
\end{array}\right)=j 2^{q-3} q\left|x_{j}-y_{j}\right|^{q-4}\left(\left|x_{j}-y_{j}\right|^{2}|x-y|^{2}+(q-2)<x_{j}-y_{j}, x-y>^{2}\right)
$$


and, on the other hand

$$
\begin{aligned}
u_{j}(x) & +v_{j}(y)=u\left(x+x_{j}\right)-u\left(x_{j}\right)-v\left(y+y_{j}\right)+v\left(y_{j}\right) \\
& -j\left|x_{j}-y_{j}\right|^{q-2}<x_{j}-y_{j}, x>+j\left|x_{j}-y_{j}\right|^{q-2}<x_{j}-y_{j}, y>.
\end{aligned}
$$

Adding and subtracting $\frac{j}{q}\left|x+x_{j}-y-y_{j}\right|^{q}-\frac{j}{q}\left|x_{j}-y_{j}\right|^{q}$, one gets

$$
\begin{aligned}
u_{j}(x) & +v_{j}(y)=\psi_{j}\left(x+x_{j}, y+y_{j}\right)-\psi_{j}\left(x_{j}, y_{j}\right)+\frac{j}{q}\left|x+x_{j}-y-y_{j}\right|^{q} \\
& -\frac{j}{q}\left|x_{j}-y_{j}\right|^{q}-j\left|x_{j}-y_{j}\right|^{q-2}<x_{j}-y_{j}, x-y>.
\end{aligned}
$$

Hence

$$
\begin{aligned}
& u_{j}(x)+v_{j}(y)-(x, y) A\left(\begin{array}{c}
x \\
y
\end{array}\right) \\
= & \psi_{j}\left(x+x_{j}, y+y_{j}\right)-\psi_{j}\left(x_{j}, y_{j}\right)+\frac{j}{q}\left|x+x_{j}-y-y_{j}\right|^{q}-\frac{j}{q}\left|x_{j}-y_{j}\right|^{q} \\
- & j\left|x_{j}-y_{j}\right|^{q-2}<x_{j}-y_{j}, x-y> \\
- & j 2^{q-3} q\left(\left|x_{j}-y_{j}\right|^{q-4}\left(\left|x_{j}-y_{j}\right|^{2}|x-y|^{2}+(q-2)<x_{j}-y_{j}, x-y>^{2}\right)\right) .
\end{aligned}
$$

Since by the definition of $\left(x_{j}, y_{j}\right)$ one has

$$
\psi_{j}\left(x+x_{j}, y+y_{j}\right)-\psi_{j}\left(x_{j}, y_{j}\right) \leq 0
$$

it is sufficient to prove that

$$
\begin{aligned}
& 0 \geq \frac{j}{q}\left|x+x_{j}-y-y_{j}\right|^{q}-\frac{j}{q}\left|x_{j}-y_{j}\right|^{q}-j\left|x_{j}-y_{j}\right|^{q-2}<x_{j}-y_{j}, x-y>+ \\
& -j 2^{q-3} q\left(\left|x_{j}-y_{j}\right|^{q-4}\left(\left|x_{j}-y_{j}\right|^{2}|x-y|^{2}+(q-2)<x_{j}-y_{j}, x-y>^{2}\right)\right)
\end{aligned}
$$

This can be obtained using the convexity inequality in claim 2 , with $\xi=$ $\frac{x_{j}-y_{j}}{\left|x_{j}-y_{j}\right|}$ and $\eta=\frac{x-y}{\left|x_{j}-y_{j}\right|}$.

Second case : Suppose that $|x| \leq \epsilon$ and $|y| \geq \epsilon$. 
One may write, using the first case for $|x| \leq \epsilon$ and $y=0$

$$
\begin{aligned}
\frac{-|y|^{2}}{\epsilon^{3}}+u_{j}(x) & \leq(x, 0) A_{j}\left(\begin{array}{l}
x \\
0
\end{array}\right)-\frac{|y|^{2}}{\epsilon^{3}} \\
& =(x, y) A_{j}\left(\begin{array}{l}
x \\
y
\end{array}\right)-(0, y) A_{j}\left(\begin{array}{l}
0 \\
y
\end{array}\right)+ \\
& -(x, 0) A_{j}\left(\begin{array}{l}
0 \\
y
\end{array}\right)-(0, y) A_{j}\left(\begin{array}{l}
x \\
0
\end{array}\right)-\frac{|y|^{2}}{\epsilon^{3}} \\
& \leq(x, y) A_{j}\left(\begin{array}{l}
x \\
y
\end{array}\right)+|| A_{j}||\left(|y|^{2}+2|y||x|\right)-\frac{|y|^{2}}{\epsilon^{3}} \\
& \leq(x, y) A_{j}\left(\begin{array}{l}
x \\
y
\end{array}\right)+\left(k_{j}-\frac{1}{\epsilon^{3}}\right)\|y\|^{2} \\
& \leq(x, y) A_{j}\left(\begin{array}{l}
x \\
y
\end{array}\right)
\end{aligned}
$$

by the choice of $\epsilon$. The case where $|x|>\epsilon$ and $|y| \leq \epsilon$ is analogous.

Third case Suppose that $|x|,|y| \geq \epsilon$. Then

$$
\frac{-|y|^{2}}{\epsilon^{3}}-\frac{|x|^{2}}{\epsilon^{3}} \leq-2\left(|| A_{j}||\right)\left(|x|^{2}+|y|^{2}\right) \leq(x, y) A_{j}\left(\begin{array}{l}
x \\
y
\end{array}\right) .
$$

We now apply Lemma 2.3 to $u_{j}, v_{j}$ with $\epsilon=\frac{1}{j}$. Hence $\left(0, X_{j}\right) \in J^{2,+} \tilde{u}_{j}(0)$, $\left(0,-Y_{j}\right) \in J^{2,-} \tilde{v}_{j}(0)$ and

$$
\left(\begin{array}{cc}
X_{j} & 0 \\
0 & Y_{j}
\end{array}\right) \leq A_{j}+\frac{1}{j} A_{j}^{2} \leq 2 j k_{j}\left(\begin{array}{cc}
I & -I \\
-I & I
\end{array}\right),
$$

in the second inequality we have used claim 1 . Noting that $J^{2,+} u_{j}(0)=\left[J^{2,+} u\left(x_{j}\right)\right]-\left(j\left|x_{j}-y_{j}\right|^{q-2}\left(x_{j}-y_{j}\right), 0\right)$, we see that

$$
\left(j\left|x_{j}-y_{j}\right|^{q-2}\left(x_{j}-y_{j}\right), X_{j}\right) \in J^{2,+} u\left(x_{j}\right) .
$$

Similarly

$$
\left(j\left|x_{j}-y_{j}\right|^{q-2}\left(x_{j}-y_{j}\right),-Y_{j}\right) \in J^{2,-} v\left(y_{j}\right) .
$$

This ends the proof of Lemma 2.1. 
Proof of claim 1

Computing $A_{j}$ one gets

$$
A_{j}=j\left(\begin{array}{cc}
D_{j}+2 D_{j}^{2} & -D_{j}-2 D_{j}^{2} \\
-D_{j}-2 D_{j}^{2} & D_{j}+2 D_{j}^{2}
\end{array}\right)
$$

and $D_{j}+2 D_{j}^{2}$ is a symmetric matrix which has a norm less than $\left\|D_{j}\right\|(1+$ $\left.2^{q-2} q(q-1)\left|x_{j}-y_{j}\right|^{q-2}\right) \leq\left\|D_{j}\right\|\left(1+j^{-1+\frac{2}{q}}\right) \leq 2\left\|D_{j}\right\|$ for $j$ large enough.

Claim 1 is a direct consequence of

Lemma 2.4 For all symmetric matrix $A$, one has

$$
-3\|A\|\left(\begin{array}{cc}
I & 0 \\
0 & I
\end{array}\right) \leq\left(\begin{array}{cc}
A & -A \\
-A & A
\end{array}\right) \leq\|A\|\left(\begin{array}{cc}
I & -I \\
-I & I
\end{array}\right)
$$

where $\|A\|$ is the norm subordinate to the Euclidean norm i.e. $\|A\|=\sup _{x,|x|=1}|A x|$ and $|x|^{2}=\sum_{i} x_{i}^{2}$.

Proof of lemma 2.4 One must prove that for all $(X, Y) \in \mathbb{R}^{2 N}$

$$
{ }^{t}(X, Y)\left(\|A\|\left(\begin{array}{cc}
I & -I \\
-I & I
\end{array}\right)-\left(\begin{array}{cc}
A & -A \\
-A & A
\end{array}\right)\right)(X, Y) \geq 0
$$

One has

$$
\begin{aligned}
{ }^{t}(X, Y)\left(\begin{array}{cc}
A & -A \\
-A & A
\end{array}\right)(X, Y) & ={ }^{t} X(A X-A Y)+{ }^{t} Y(-A X+A Y) \\
& ={ }^{t} X A X-{ }^{t} X A Y-{ }^{t} Y A X+{ }^{t} Y A Y \\
& ={ }^{t}(X-Y) A(X-Y) \\
& \leq\|A\| \|^{t}(X-Y)(X-Y) \\
& ={ }^{t}(X, Y)\|A\|\left(\begin{array}{cc}
I & -I \\
-I & I
\end{array}\right)(X, Y)
\end{aligned}
$$

Proof of Claim 2

To prove (2.4), let us define on $[0,1]$ the function $f$ :

$f(t)=\frac{1}{q}|\xi+t \eta|^{q}-\frac{1}{q}-t<\xi, \eta>-t^{2} 2^{q-3} q\left(|\eta|^{2}+(q-2)<\xi, \eta>^{2}\right)$. 
One observes that $f(0)=0$,

$f^{\prime}(t)=|\xi+t \eta|^{q-2}<\xi+t \eta, \eta>-<\xi, \eta>-q 2^{q-2} t\left(|\eta|^{2}+(q-2)<\xi, \eta>^{2}\right)$.

One has $f^{\prime}(0)=0$ and

$$
\begin{aligned}
f^{\prime \prime}(t) & =(q-2)|\xi+t \eta|^{q-4}<\xi+t \eta, \eta>^{2} \\
& +|\xi+t \eta|^{q-2}|\eta|^{2}-2^{q-2} q\left(|\eta|^{2}+(q-2)<\xi, \eta>^{2}\right) \leq 0 .
\end{aligned}
$$

Indeed,

$$
\begin{aligned}
& (q-2)|\xi+t \eta|^{q-4}<\xi+t \eta, \eta>^{2}+|\xi+t \eta|^{q-2}|\eta|^{2} \\
= & |\xi+t \eta|^{q-4}\left(<\xi, \eta>^{2}(q-2)+t^{2}|\eta|^{4}(q-2)+2(q-2) t<\xi, \eta>|\eta|^{2}\right. \\
+ & \left.|\eta|^{2}+t^{2}|\eta|^{4}+2 t<\xi, \eta>|\eta|^{2}\right) \\
= & |\xi+t \eta|^{q-4}\left((q-2)<\xi, \eta>^{2}+|\eta|^{2}\left((q-1)\left(t^{2}|\eta|^{2}+2 t<\xi, \eta>\right)+1\right)\right) \\
\leq & 2^{q-4}\left(<\xi, \eta>^{2}(q-2)+|\eta|^{2}(3 q-2)\right) \\
\leq & 2^{q-2} q\left(<\xi, \eta>^{2}(q-2)+|\eta|^{2}\right) .
\end{aligned}
$$

Finally $f^{\prime}$ is negative on $[0,1]$ and $f$ as well. This proves (2.4).

We now state and prove a strong maximum principle when there is no explicit dependence on $u$ in the equation.

Proposition 2.2 Let $\Omega$ be a bounded open set in $\mathbb{R}^{N}$. Suppose that $F$ satisfies 1 and 3 with $\alpha=\beta$. Let $u$ in $\mathcal{C}(\bar{\Omega}), u \geq 0$ in $\Omega$ be a super-solution of $F(x, \nabla u, \nabla \nabla u)=0$. Then, either $u$ is strictly positive inside $\Omega$, or $u$ is identically zero.

Proof.

Using the inequality satisfied by $F$ in its definition, let us recall, using Remark 2.1, that

$$
\begin{aligned}
F(x, p, M) & \geq|p|^{\alpha}\left(\lambda \operatorname{tr}(M)^{+}-\Lambda \operatorname{tr}(M)^{-}\right) \\
& :=G(p, M)
\end{aligned}
$$


hence it is sufficient to prove the proposition when $u$ is a super-solution of $G=0$. $G$ does not depend on $x$ and it satisfies the hypothesis of Theorem 2.1 .

Let us suppose that $x_{0}$ is some point inside $\Omega$ on which $u\left(x_{0}\right)=0$. Following e.g. Vasquez [17], one can assume that on the ball $\left|x-x_{1}\right|=$ $\left|x-x_{0}\right|=R, x_{0}$ is the only point on which $u$ is zero and that $B\left(x_{1}, \frac{3 R}{2}\right) \subset \Omega$. Let $u_{1}=\inf _{\left|x-x_{1}\right|=\frac{R}{2}} u>0$, by the continuity of $u$. Let us construct a subsolution on the annulus $\frac{R}{2} \leq\left|x-x_{1}\right|=\rho<\frac{3 R}{2}$.

Let us recall that if $\phi(\rho)=e^{-c \rho}$, the eigenvalues of $D^{2} \phi$ are $\phi^{\prime \prime}(\rho)$ of multiplicity 1 and $\frac{\phi^{\prime}}{\rho}$ of multiplicity $\mathrm{N}-1$.

Then take $c$ such that

$$
c>\frac{2(N-1) \Lambda}{R \lambda}
$$

If $c$ is as above, let $a$ be chosen such that

$$
a\left(e^{-c R / 2}-e^{-c R}\right)=u_{1}
$$

and define $v(x)=a\left(e^{-c \rho}-e^{-c R}\right)$. The function $v$ is a strict sub-solution of $G=0$. Furthermore

$$
\left\{\begin{array}{c}
v \leq u \quad \text { on }\left|x-x_{1}\right|=\frac{R}{2} \\
v \leq 0 \leq u \quad \text { on }\left|x-x_{1}\right|=\frac{3 R}{2}
\end{array}\right.
$$

hence $u \geq v$ everywhere on the boundary of the annulus. Using the comparison principle Theorem 2.1 for the operator $G, u \geq v$ everywhere on the annulus, and then $v$ is a test function for $u$ on the test point $x_{0}$. One must then have since $u$ is a super-solution and $D v\left(x_{0}\right) \neq 0$,

$$
-G\left(D v\left(x_{0}\right), D^{2} v\left(x_{0}\right)\right) \geq 0
$$

which clearly contradicts the definition of $v$. Finally $u$ cannot be zero inside $\Omega$. 
Remark 2.4 A Hopf's property

Using the same construction and assuming that $x_{0} \in \partial \Omega$, replacing the previous annulus by its "half part" $\frac{R}{2} \leq\left|x-x_{1}\right| \leq R$ and using the comparison principle, since $v=0$ on $\left|x-x_{1}\right|=R, D v \neq 0$ in $\Omega$ and $v \leq u$ on the other boundary of the annulus, one gets that

$$
u(x) \geq a\left(e^{-c \rho}-e^{-c R}\right)
$$

and then taking $x=x_{0}-h \vec{n}$ and letting $h>0$ go to zero, one gets

$$
\frac{u(x)-u\left(x_{0}\right)}{h} \geq a \frac{e^{-c R+c h}-e^{-c R}}{h} \rightarrow a c e^{-c R}
$$

This, for example, implies that $D u\left(x_{0}\right) \neq 0$ when $u$ is $\mathcal{C}^{1}$.

\section{Liouville's Theorem}

As mentioned in the introduction we consider now $F(x, p, X)$ continuous and satisfying conditions 1,3 for any $x \in \mathbb{R}^{n}$. In all the section we will suppose that $\alpha=\beta$ in condition 3. Finally we will denote by $\mu$ the real number

$$
\mu=\frac{\Lambda}{\lambda}(N-1)-1 .
$$

Using the comparison's Theorem 2.1 and the strong maximum principle in Proposition 2.2 obtained in the previous section we want to prove the following

Theorem 3.1 Suppose that $u \in \mathcal{C}\left(\mathbb{R}^{N}\right)$ is a nonnegative viscosity solution of

$$
-F\left(x, \nabla u, D^{2} u\right) \geq h(x) u^{\bar{q}} \text { in } \mathbb{R}^{N}
$$

with $h$ satisfying

$$
h(x)=a|x|^{\gamma} \text { for }|x| \text { large, } a>0 \text { and } \gamma>-(\alpha+2) .
$$

Suppose that

$$
0<\bar{q} \leq \frac{1+\gamma+(\alpha+1)(\mu+1)}{\mu}
$$

then $u \equiv 0$. 
Now recalling Remark 2.1, condition 3 with $\alpha=\beta$ implies that if $u$ is a solution of (3.1) then it is also a solution of

$$
-\mathcal{M}_{\lambda, \Lambda}^{-}\left(D^{2} u\right)|\nabla u|^{\alpha} \geq h(x) u^{\bar{q}}
$$

Therefore in the proof of Theorem 3.1 we shall consider this inequation, using the same notation $F$ for its left hand side. Before giving the proof of Theorem 3.1 Let us define $m(r)=\inf _{x \in B_{r}} u(x)$. Let us note that if $u$ is not identically zero and satisfies (3.1), the strict maximum principle in Proposition 2.2 implies that $m(r)>0$.

We now prove the following Hadamard type inequality

Proposition 3.1 Let $u$ be a viscosity solution of $-F\left(x, \nabla u, D^{2} u\right) \geq 0$ and $u \geq 0$, which is not identically zero. For any $0<R_{1}<r<R_{2}$ :

$$
m(r) \geq \frac{m\left(R_{1}\right)\left(r^{-\mu}-R_{2}^{-\mu}\right)+m\left(R_{2}\right)\left(R_{1}^{-\mu}-r^{-\mu}\right)}{R_{1}^{-\mu}-R_{2}^{-\mu}} .
$$

Proof. This is immediate using the comparison principle Theorem 2.1 with $b=0$ in $B_{R_{2}} \backslash B_{R_{1}}$ between the function $u$ and the function $\phi$ defined by $\phi(x)=g(|x|)$ with $g(r)=C_{1} r^{-\mu}+C_{2}$ where $C_{1}$ and $C_{2}$ are chosen such that $\phi(x)=m\left(R_{1}\right)$ on $\partial B_{R_{1}}$ and $\phi(x)=m\left(R_{2}\right)$ on $\partial B_{R_{1}}$. Using Remark 2.1, we can apply the comparison principle Theorem 2.1 in $B_{r_{2}} \backslash B_{r_{1}}$ between $u$ and $\phi$. And this gives precisely (3.3).

Corollary 3.1 Suppose that $u$ satisfies the assumptions in Proposition 3.1. Then, for $r \geq R_{1}$ :

$$
m(r) \geq \frac{m\left(R_{1}\right) r^{-\mu}}{r_{1}^{-\mu}} .
$$

Just observe that since $\mu>0$, by letting $R_{2}$ tend to infinity in (3.3) we obtain the above inequality.

Corollary 3.2 We still assume that u satisfies the assumptions in Proposition 3.1 Suppose that $1 \leq r \leq r_{1}$ and $r_{1} \geq 2$. Then

$$
m(r)-m\left(r_{1}\right) \geq(m(1)-m(2))\left(r^{-\mu}-r_{1}^{-\mu}\right) .
$$

As a consequence for $0 \leq \theta \leq \frac{1}{2}$

$$
m\left(r_{1}(1-\theta)\right)-m\left(r_{1}\right)>(m(1)-m(2)) r_{1}^{-\mu} \theta \mu .
$$


Proof

We use the inequality

$$
m(r)-m\left(R_{2}\right) \geq \frac{m\left(R_{1}\right)-m\left(R_{2}\right)}{R_{1}^{-\mu}-R_{2}^{-\mu}}\left(r^{-\mu}-R_{2}^{-\mu}\right)
$$

which is equivalent to (3.3) in Proposition 3.1 with $R_{1}=1$, and $R_{2}=r_{1} \geq 2$ and $m\left(R_{2}\right)=m\left(r_{1}\right) \leq m(2)$ to obtain (3.4).

We then use the mean value theorem and the fact that $\left(1-\theta^{\prime}\right)^{-(\mu+1)}>1$ when $1>\theta^{\prime}>0$.

Proof of Theorem 3.1.

We suppose by contradiction that $u \not \equiv 0$ in $\mathbb{R}^{N}$, but since $u \geq 0$ and $u$ is a super-solution in the viscosity sense, using Proposition 2.2 one has $u>0$. We denote by $C$ the constant

$$
C=\frac{m(1)-m(2)}{\left(1-2^{-\mu}\right)} \frac{\mu}{m\left(r_{1}\right) r_{1}^{\mu}}
$$

Let $1<r_{1} \leq \frac{R}{2}$, define $g(r)=m\left(r_{1}\right)\left\{1-\frac{C\left(r-r_{1}\right)}{\left(R-r_{1}\right)}-\frac{\left[\left(r-r_{1}\right)^{+}\right]^{3}}{\left(R-r_{1}\right)^{3}}\right\}$. Let $\zeta(x)=g(|x|)$. Clearly for $|x| \geq R, \zeta(x) \leq 0<u(x)$. On the other hand there exists $\tilde{x}$ such that $|\tilde{x}|=r_{1}$ and $u(\tilde{x})=\zeta(\tilde{x})$.

Let us observe that the definition of $C$ implies that $u-\zeta$ has a local minimum on $\left[r_{1}, R\right]$. For this, one proves that for $\theta \leq \frac{1}{2}$, for $x$ such that $r_{1} \geq|x| \geq \frac{r_{1}}{2}, u(x)>\zeta(x)$. Indeed, for such $x,|x|=r_{1}(1-\theta)$

$$
\begin{aligned}
\zeta(x) & =g\left(r_{1}(1-\theta)\right) \\
& =m\left(r_{1}\right)+\frac{m(1)-m(2)}{\left(1-2^{-\mu}\right)} r_{1}^{-\mu} \frac{r_{1} \theta \mu}{\left(R-r_{1}\right)} \\
& \leq m\left(r_{1}\right)+\frac{m(1)-m(2)}{\left(1-2^{-\mu}\right)} r_{1}^{-\mu} \theta \mu \\
& <m\left(r_{1}(1-\theta)\right) \\
& \leq u(x) .
\end{aligned}
$$

Hence a local minimum of $u(x)-\zeta(x)$ occurs for some $\bar{x}$ such that $|\bar{x}|=\bar{r}$ with $r_{1} \leq \bar{r}<R$. 
Let $|x|=r$, it is an easy computation to see that for $r \geq r_{1}$

$$
g^{\prime}(r)=-m\left(r_{1}\right)\left(\frac{C}{R-r_{1}}+3 \frac{\left(r-r_{1}\right)^{2}}{\left(R-r_{1}\right)^{3}}\right)
$$

and

$$
g^{\prime \prime}(r)=-m\left(r_{1}\right)\left(\frac{6\left(r-r_{1}\right)}{\left(R-r_{1}\right)^{3}}\right)
$$

and then

$$
\begin{aligned}
-F\left(x, \nabla \zeta, D^{2} \zeta(x)\right) & \leq-\lambda|\nabla \zeta|^{\alpha}(\Delta \zeta) \\
& \leq-\lambda|\nabla \zeta|^{\alpha}\left(g^{\prime \prime}(r)+\left(\frac{N-1}{r}\right) g^{\prime}(r)\right) \\
& \leq \lambda m\left(r_{1}\right)^{\alpha+1}\left|\frac{C+3}{\left(R-r_{1}\right)}\right|^{\alpha}\left(\frac{6}{\left(R-r_{1}\right)^{2}}+\left(\frac{N-1}{r}\right) \frac{C+3}{\left(R-r_{1}\right)}\right) \\
& \leq m\left(r_{1}\right)^{\alpha+1} \frac{C^{\prime}}{\left(R-r_{1}\right)^{\alpha+2}}
\end{aligned}
$$

using $r \geq r_{1} \geq R-r_{1}$ and for some universal constant $C^{\prime}$. Since $\nabla \zeta(\bar{x}) \neq 0$, using the definition of viscosity solution

$$
h(\bar{x}) u^{q}(\bar{x}) \leq-F\left(\bar{x}, \nabla \zeta(\bar{x}), D^{2} \zeta(\bar{x})\right) .
$$

We choose $r_{1}$ and $R$ sufficiently large in order that $h(x) \geq a|x|^{\gamma}$ for $|x| \geq \frac{R}{2}$. Combining this with (3.5), we obtain

$$
a \bar{r}^{\gamma} m(\bar{r})^{\bar{q}} \leq a \bar{r}^{\gamma} u^{\bar{q}}(\bar{x}) \leq C^{\prime} m\left(r_{1}\right)^{\alpha+1}\left(R-r_{1}\right)^{-(\alpha+2)} .
$$

Since $m$ is decreasing the previous inequality becomes

$$
m(R) \leq C^{\prime \prime} m\left(r_{1}\right)^{\frac{\alpha+1}{\bar{q}}} \bar{r} \frac{-\gamma}{\bar{q}}\left(R-r_{1}\right)^{-\frac{\alpha+2}{\bar{q}}} .
$$

Now we choose $r_{1}=\frac{R}{2}$, we use Corollary 3.1 and finally we get

$$
m(R) \leq C m(R)^{\frac{(\alpha+1)}{\bar{q}}} R^{\frac{-(\alpha+2+\gamma)}{\bar{q}}} .
$$


First we will suppose that $\bar{q} \leq \alpha+1$; hence, using the monotonicity of $m(R)$, the above inequality becomes

$$
R^{\frac{\alpha+2+\gamma}{\bar{q}}} \leq C^{\prime \prime} m(R)^{\frac{(\alpha+1)}{\bar{q}}-1} \leq C^{\prime \prime} u(0)^{\frac{(\alpha+1)}{\bar{q}}-1} .
$$

Since we are supposing that $\alpha+2+\gamma \geq 0$, we get a contradiction. This concludes this case.

Now suppose that $\bar{q}>\alpha+1$, this implies that (3.6) becomes

$$
m(R) R^{\mu} \leq C^{\prime \prime} R^{\mu-\frac{(\alpha+2+\gamma)}{\bar{q}-(\alpha+1)}}
$$

If $\bar{q}<\frac{1+\gamma+(\alpha+1)(\mu+1)}{\mu}$ then $\mu-\frac{(\alpha+2+\gamma)}{\bar{q}-(\alpha+1)}<0$. We have reached a contradiction since the right hand side of (3.7) tends to zero for $R \rightarrow+\infty$ while the left hand side is an increasing positive function as seen in Corollary 3.1.

This concludes the proof of this case.

We now treat the case $\bar{q}=\frac{1+\gamma+(\alpha+1)(\mu+1)}{\mu}$. Let us remark that for this choice of $\bar{q}$ we have that for some $C_{1}>0, c>0$ and $r>r_{1}>0$, with $r_{1}$ large enough :

$$
-F\left(x, \nabla u, D^{2} u\right) \geq a r^{\gamma} u^{\bar{q}} \geq C_{1} r^{-(\mu+1)(\alpha+1)-1} .
$$

We choose $\psi(x)=g(|x|)$ with

$$
g(r)=\gamma_{1} r^{-\mu} \log ^{\nu} r+\gamma_{2}
$$

where $\gamma_{1}$ and $\gamma_{2}$ are two positive constants such that for some $r_{1}>1$ and some $r_{2}>r_{1}$ :

$$
\begin{aligned}
& m\left(r_{2}\right)=g\left(r_{2}\right), \\
& m\left(r_{1}\right) \geq g\left(r_{1}\right),
\end{aligned}
$$

while $\nu$ is a positive constant to be chosen later. It is easy to see that

$$
\begin{aligned}
& |\nabla \psi|^{\alpha} \mathcal{M}_{\lambda, \Lambda}^{-}\left(D^{2} \psi\right) \\
= & \left|-\mu+\frac{\nu}{\log r}\right|^{\alpha}\left|\gamma_{1}\right|^{\alpha+1} r^{-(\mu+1) \alpha} \log ^{\nu \alpha} r\left[r^{-(\mu+2)} \log ^{\nu} r \mu(\lambda(\mu+1)-(N-1) \Lambda)\right. \\
& \left.-\lambda \mu \nu r r^{-(\mu+2)} \log ^{\nu-1}+\lambda \nu(\nu-1) r^{-(\mu+2)} \log ^{\nu-2} r\right] \\
\leq & -C r^{-(\mu+1)(\alpha+1)-1}(\log r)^{\nu \alpha+\nu-1} .
\end{aligned}
$$


We have used the fact that $\lambda(\mu+1)-(N-1) \Lambda=0$.

We can choose $\nu>0$ such that $\nu \alpha+\nu-1<0$. Using 3.8 this allows us to get

$$
\begin{aligned}
-F\left(x, \nabla u, D^{2} u\right) & \geq C r^{-(\mu+1)(\alpha+1)-1} \\
& \geq C r^{-(\mu+1)(\alpha+1)-1}(\log r)^{\nu \alpha+\nu-1} \geq-|\nabla \psi|^{\alpha} \mathcal{M}_{\lambda, \Lambda}^{-}\left(D^{2} \psi\right) .
\end{aligned}
$$

Since $u \geq \psi$ on the boundary of $B_{r_{2}} \backslash B_{r_{1}}$, one obtains by the comparison principle that $u \geq \psi$ everywhere in $B_{r_{2}} \backslash B_{r_{1}}$.

When $r_{2}$ goes to infinity it is easy to see that $\gamma_{2} \rightarrow 0$, and we obtain

$$
u(x) \geq c|x|^{-\mu} \log ^{\nu}|x|
$$

for $|x| \geq r_{1}$. This implies that

$$
m(r) \geq c r^{-\mu} \log ^{\nu} r
$$

for $r>r_{1}$. We have reached a contradiction since

$$
m(r) \leq C r^{-\mu}
$$

Hence $u \equiv 0$. This concludes the proof of Theorem 3.1.

\section{References}

[1] H.Berestycki, I. Capuzzo Dolcetta, L. Nirenberg Problèmes Elliptiques indéfinis et Théorème de Liouville non-linéaires, C. R. Acad. Sci. Paris Sr. I Math. 317 (1993), no. 10, 945-950.

[2] H.Berestycki, I. Capuzzo Dolcetta, L. Nirenberg, Superlinear indefinite elliptic problems and nonlinear Liouville theorems. Topol. Methods Nonlinear Anal. 4 (1994), no. 1, 59-78.

[3] I. Birindelli, F. Demengel Some Liouville Theorems for the p-Laplacian Elec. J. Differential Equations, Conference 08, 2002.

[4] I. Birindelli, E. Mitidieri, Liouville theorems for elliptic inequalities and applications. Proc. Roy. Soc. Edinburgh Sect. A 128 (1998), no. 6, 12171247. 
[5] L. Caffarelli, X. Cabré Fully-nonlinear equations Colloquium Publications 43, American Mathematical Society, Providence, RI,1995.

[6] Y.G. Chen, Y. Giga, S. Goto Uniqueness and existence of viscosity solutions of generalized mean curvature flow equations Journal of Differential Geometry, 33, (1991), 749-786.

[7] M.G. Crandall,H. Ishii, P.L. Lions User's guide to viscosity solutions of second order partial differential equations. Bull. Amer. Math. Soc. (N.S.) 27 (1992), no. 1, 1-67.

[8] A. Cutri, F. Leoni On the Liouville property for fully-nonlinear equations Annales de l'Institut H. Poincaré, Analyse non-linéaire, (2000), 219-245.

[9] C. Evans, J. Spruck Motion of level sets by mean curvature Journal of Diff. Geom. 33, (1991), 635-681.

[10] B. Gidas, Symmetry properties and isolated singularities of positive solutions of nonlinear elliptic equations, Nonlinear Partial Differential equations in engineering and applied sciences, Eds. R. Sternberg, A. Kalinowski and J. Papadakis, Proc. Conf. Kingston, R.I. 1979, Lect. Notes on pure appl. maths, 54, Decker, New York, 1980, 255-273.

[11] H. Ishii Viscosity solutions of non-linear partial differential equations Sugaku Expositions vol 9, (1996).

[12] R. Jensen The maximum principle for viscosity solutions of fully nonlinear second order partial differential equations Arch. Rational Mech. Anal. 101 (1988) 1-27.

[13] R. Jensen, P.L. Lions, T. Souganidis A uniqueness result for viscosity solutions of second order fully-nonlinear partial differential equations Proc. Amer.Math. Soc. 102 (1988), 975-978.

[14] P. Juutinen, P. Lindquist, J. Manfredi On the equivalence of viscosity solutions and weak solutions for a quasi linear equation. SIAM J. Math. Anal. 33 (2001), no. 3, 699-717. 
[15] E. Mitidieri, S. Pohozaev Absence of positive solutions for quasilinear elliptic problems in $R^{N}$. (Russian) Tr. Mat. Inst. Steklova 227 (1999), Issled. po Teor. Differ. Funkts. Mnogikh Perem. i ee Prilozh. 18, 192-222; translation in Proc. Steklov Inst. Math. 1999, no. 4 (227), 186-216.

[16] J. Serrin,H. Zou Cauchy-Liouville and universal boundedness theorems for quasi-linear elliptic equations.Acta Math. 189 (2002), no. 1, 79-142.

[17] J.L. Vasquez A strong maximum principle for some quasilinear elliptic equations, Appl. Math. Optim. 12: 191-202, (1984).

[18] J. Wigniolle A strong maximum principle for fully non linear degenerate operators Prépublications de l'université de Cergy-Pontoise.

Isabeau Birindelli

Università di Roma "La Sapienza"

Piazzale Aldo moro, 5

00185 Roma, Italy

e mail: isabeau@mat.uniroma1.it

Françoise Demengel

Université de Cergy Pontoise,

Site de Saint-Martin, 2 Avenue Adolphe Chauvin

95302 Cergy-Pontoise, France

e-mail: francoise.demengel@math.u-cergy.fr 